\title{
Efficacy of pain control with topical lidocaine-epinephrine- tetracaine during laceration repair with tissue adhesive in children: a randomized controlled trial
}

\author{
Stuart Harman MD, Roger Zemek MD, Mary Jean Duncan MD, Yvonne Ying MD, William Petrcich MSc
}

\begin{abstract}
Background: Some children feel pain during wound closures using tissue adhesives. We sought to determine whether a topically applied analgesic solution of lidocaineepinephrine-tetracaine would decrease pain during tissue adhesive repair.
\end{abstract}

\begin{abstract}
Methods: We conducted a randomized, placebocontrolled, blinded trial involving 221 children between the ages of 3 months and 17 years. Patients were enrolled between March 2011 and January 2012 when presenting to a tertiary-care pediatric emergency department with lacerations requiring closure with tissue adhesive. Patients received either lidocaine-epinephrine-tetracaine or placebo before undergoing wound closure. Our primary outcome was the pain rating of adhesive application according to the colour Visual Analogue Scale and the Faces Pain Scale Revised. Our secondary outcomes were physician ratings of difficulty of wound closure and wound hemostasis, in addition to their prediction as to which treatment the patient had received.
\end{abstract}

Results: Children who received the analgesic before wound closure reported less pain (median 0.5, interquartile range [IQR] 0.251.50) than those who received placebo (median 1.00, IQR 0.38-2.50) as rated using the colour Visual Analogue Scale $(p=0.01)$ and Faces Pain Scale - Revised (median 0.00, IQR 0.00-2.00, for analgesic v. median 2.00, IQR 0.00-4.00, for placebo, $p<0.01$ ). Patients who received the analgesic were significantly more likely to report having or to appear to have a pain-free procedure (relative risk [RR] of pain $0.54,95 \%$ confidence interval [CI] 0.37-0.80). Complete hemostasis of the wound was also more common among patients who received lidocaine-epinephrine-tetracaine than among those who received placebo (78.2\% v. $59.3 \%, p=0.008)$.

Interpretation: Treating minor lacerations with lidocaine-epinephrine-tetracaine before wound closure with tissue adhesive reduced ratings of pain and increased the proportion of pain-free repairs among children aged 3 months to 17 years. This low-risk intervention may benefit children with lacerations requiring tissue adhesives instead of sutures. Trial registration: ClinicalTrials.gov, no. PR 6138378804.
$\mathrm{M}$ inor laceration repair with tissue adhesive, or "skin glue," is common in pediatrics. Although less painful than cutaneous sutures, ${ }^{1}$ tissue adhesives polymerize through an exothermic reaction that may cause a burning, painful sensation. Pain is dependent on the specific formulation of the adhesive used and the method of application. One study of different tissue adhesives reported $23.8 \%-40.5 \%$ of participants feeling a "burning sensation", ${ }^{2}$ whereas another study reported "pain" in $17.6 \%-44.1 \%$ of children. ${ }^{3}$ The amounts of adhesive applied, method of application and individual patient characteristics can also influence the feeling of pain. ${ }^{3.4}$ Because tissue adhesives polymerize on contact with moisture, ${ }^{4,5}$ poor wound hemostasis has the potential to cause premature setting of the adhesive, leading to less efficient and more painful repairs. ${ }^{6}$
Preventing procedural pain is a high priority in pediatric care. ${ }^{7}$ Inadequate analgesia for pediatric procedures may result in more complicated procedures, increased pain sensitivity with future procedures $^{8}$ and increased fear and anxiety of medical experiences persisting into adulthood. ${ }^{9}$ A practical method to prevent pain during laceration repairs with tissue adhesive would have a substantial benefit for children.

A topically applied analgesic solution containing lidocaine-epinephrine-tetracaine with vasoconstrictive properties provides safe and effective pain control during wound repair using sutures. ${ }^{10}$ A survey of pediatric emergency fellowship directors in the United States reported that $76 \%$ of respondents use this solution or a similar solution when suturing $3-\mathrm{cm}$ chin lacerations in toddlers. ${ }^{11}$ However, in a hospital chart review, this
Competing interests: None declared.

This article has been peer reviewed.

Correspondence to: Stuart Harman, sharman@cheo.on.ca

CMAJ 2013. DOI:10.1503 /cmaj.130269 
solution was used in less than half of tissue adhesive repairs, the remainder receiving either local injection of anesthetic or no pain control. ${ }^{12}$ Reluctance to use lidocaine-epinephrine-tetracaine with tissue adhesive may be due to the perception that it is not worth the minimum 20-minute wait required for the analgesic to take effect ${ }^{13}$ or to a lack of awareness that tissue adhesives can cause pain.

We sought to investigate whether preapplying lidocaine-epinephrine-tetracaine would decrease pain in children during minor laceration repair using tissue adhesive.

\section{Methods}

\section{Design and setting}

We conducted a randomized, placebocontrolled, blinded trial. From March 2011 to January 2012, we recruited participants from a tertiary-care, academic, pediatric emergency department that reported 66000 visits and 1300 laceration repairs using tissue adhesive per year. Health Canada approved this regulated phase II clinical trial for the use of lidocaineepinephrine-tetracaine, and the Children's Hospital of Eastern Ontario Research Ethics Board approved the study.

\section{Participants}

Children aged 3 months to 17 years (inclusive) were eligible for the study if they had a laceration of less than $3 \mathrm{~cm}$ in length on the face, torso, trunk or extremities that was deemed appropriate for tissue adhesive repair by their treating physicians. We excluded children whose wounds required débridement or suturing before a first glue attempt, as well as children with wounds caused by an animal (or human) bite or scratch; wounds on the ear, fingers or toes; wounds crossing joint lines or the mucocutaneous junction, or in areas of concentrated hair such as the scalp or eyebrow; puncture or stellate crush wounds; children with a known history of keloid formation or a known allergy to cyanoacrylates; and children taking medications known to impair wound healing or hemostasis, including oral steroids, immunostimulants or anticoagulants.

Potential participants underwent screening for study inclusion in the waiting room between the hours of 1000 and 2200. Patients aged 14 years and older provided written informed consent; for children less than 14 years of age, we obtained parental or legal guardian consent and patient assent. Consent for a 2-week follow-up telephone survey was requested but was not required for participants to be included in randomization and primary data collection.

\section{Interventions}

Identical opaque envelopes containing syringes with $3 \mathrm{~mL}$ of lidocaine-epinephrine-tetracaine or placebo gel were prepared and sealed by staff in the hospital pharmacy and stored in the emergency department. Envelopes were sequenced using computer-generated randomization, coded for later identification and used by nurses in a predetermined order. The active drug and the placebo, with identity known only to pharmacy staff, were identical in appearance, volume, weight and odour. Nurses unaware of group assignment used tape or film dressing to affix cotton balls soaked with $3 \mathrm{~mL}$ of study gel to each wound. Study gel remained on wounds for a minimum of 45 minutes to ensure effectiveness. ${ }^{14}$ Study gel was removed by treating physicians at the time of wound repair, not longer than 120 minutes after application, as per protocol.

Treating physicians repaired lacerations using $n$-butyl- 2 cyanoacrylate tissue adhesive as per the usual technique. All levels of residents, fellows and staff physicians were permitted to repair wounds.

\section{Outcome measures}

Our primary outcome was the amount of pain reported during the application of tissue adhesive using the validated 10-unit colour Visual Analogue Scale. ${ }^{15,16}$ Participants aged 7 years and older rated their own pain; parents or guardians of younger participants rated their children's perceived pain. Pain was also rated by patients, parents or guardians using the validated 10-point Faces Pain Scale - Revised, ${ }^{15,17}$ a recommended adjunct to the colour Visual Analogue Scale for school-aged children. ${ }^{18,19}$

Our secondary outcome measures at the time of wound closure included physician rating of difficulty of repair on a 100 -mm visual analogue scale $(0=$ easiest repair; $100=$ most difficult repair), physician rating of wound hemostasis achieved before repair (4-point Likert scale) and physician prediction of active drug or placebo application. No previously validated tools for these secondary measures exist, although the same scale for difficulty of repair has been used in a previous study of tissue adhesives. ${ }^{20}$

Two weeks after wound repair, participants were contacted by telephone and asked about unscheduled follow-up visits and their overall satisfaction.

\section{Statistical analysis}

Using previously published pain scores for tissue adhesive, ${ }^{20}$ we calculated a desired sample size of 115 participants in each of the study arms to capture an improvement of 1 unit on the colour Visual Analogue Scale with a $p$ value of 0.05 and power of $80 \%$. Ten percent of a scale's range (e.g., 1 unit 
on a 10-unit scale) is the smallest change considered clinically significant in pain scales. ${ }^{15,21}$

We summarized baseline characteristics as means and standard deviations, medians and interquartile ranges (IQRs) or frequencies and percentages, as appropriate. We compared scores for the treatment and placebo groups from the colour Visual Analogue Scale and Faces Pain Scale - Revised using 2-sided Wilcoxon ranksum tests. We used $\chi^{2}$ tests for association for categorical variables. We calculated relative risks (RRs) and 95\% confidence intervals (CIs) of reporting pain and, where needed, CochranMantel-Haenszel estimates to adjust for age. We used a generalized linear model to assess the effects of age (dichotomized as $<7 \mathrm{yr}$ v. $\geq 7 \mathrm{yr}$ ) and group (active drug or placebo) on colour
Visual Analogue Scale score. We used a $\chi^{2}$ test to evaluate any association between dichotomized age and painful or pain-free wound closure as assessed by the Faces Pain Scale — Revised. We applied Bonferroni adjustment to establish a more conservative cut-off for significance for multiple comparators (hemostasis; $p=0.0125$ ). ${ }^{22}$ Analyses were performed using SAS version 9.2.

\section{Results}

We identified 1099 patients (Figure 1). At screening, 221 participants met our inclusion criteria, consented to at least the primary outcome data collection process and were enrolled in the study (Table 1). Of the patients initially enrolled in the study, we subsequently excluded 8 from the treat-
Excluded $n=8$

- Ineligible postallocation $n=4$

- Consent withdrawn $n=1$

- Multiple lacerations $n=1$

- Location of laceration

ineligible $n=1$

- Laceration $>3 \mathrm{~cm} \quad n=1$

- Laceration not repaired with tissue adhesive $n=4$

- Sutured $n=3$

- No primary closure required $n=1$

Patients for whom consent was provided and who were reached

for follow-up via telephone $n=85$
Patients assessed for eligibility $n=1099$
Excluded $n=878$

- Did not meet inclusion criteria $n=817$ * - Laceration not appropriate $n=368$

- Animal bite $n=53$

- Multiple lacerations $n=27$

- Patients taking incompatible medications $n=4$

- Laceration $>3 \mathrm{~cm} \quad n=46$

- Laceration on excluded location $n=140$

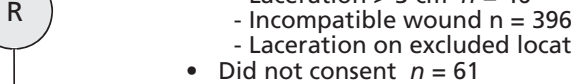

- Incompatible wound
- Laceration on excluded
- Did not consent $n=61$

Figure 1: Flow of patients through the trial. LET = lidocaine-epinepherine-tetracaine. *Some patients met more than 1 exclusion criteria. 
ment group and 10 from the placebo group from our analysis of the primary outcome, because we realized after enrolment that the patient was in fact ineligible (e.g., the treating physician decided to suture the laceration rather than use tissue adhesive), the patient had withdrawn from the study or data were missing.

\section{Primary outcome}

Children receiving lidocaine-epinephrinetetracaine reported less pain than those who received placebo, based on colour Visual Analogue Scale scores (median 0.50, IQR 0.25-1.50 for treatment group; median 1.00, IQR 0.38-2.50 for placebo group; Wilcoxon rank-sum test $p=0.01$ ) (Table 2). We found similar results when pain was reported using the Faces Pain Scale - Revised (median 0.00, IQR 0.00-2.00 for the treatment group; median 2.00, IQR $0.00-4.00$ for the placebo group; Wilcoxon rank-sum test $p<0.01$ ) (Table 2).

Pretreatment with lidocaine-epinephrinetetracaine significantly increased the proportion of pain-free procedures: $51.6 \%$ of children receiving the analgesic reported "no pain" according to the Faces Pain Scale - Revised, compared with $28.3 \%$ of those receiving placebo (RR $0.54,95 \%$ CI 0.37-0.80). This difference remained significant after controlling for age $(<7 \mathrm{yr}$. $\geq 7 \mathrm{yr})$ (RR $0.55,95 \%$ CI $0.38-0.82$ ) and for level of physician training (RR 0.56, 95\% CI 0.38-0.84). In a planned analysis, we found that for a multinomial model of colour Visual Analogue Scale score, allocation was significant $(p=0.03)$; categorized age

Table 1: Comparison of demographic and baseline characteristics of pediatric patients with lacerations repaired using tissue adhesive between the treatment and control groups

\begin{tabular}{|c|c|c|}
\hline \multirow[b]{2}{*}{ Characteristic } & \multicolumn{2}{|c|}{ Study group, no. $(\%)^{*}$} \\
\hline & $\begin{array}{l}\text { Treatment } \\
\quad n=105\end{array}$ & $\begin{array}{c}\text { Placebo } \\
n=98\end{array}$ \\
\hline Age, yr, mean \pm SD & $5.07 \pm 3.94$ & $4.21 \pm 3.62$ \\
\hline Female sex & $40(38.1)$ & $42(42.9)$ \\
\hline \multicolumn{3}{|l|}{ Location of laceration } \\
\hline Face & $100(95.2)$ & $92(94.0)$ \\
\hline Torso & $1(0.9)$ & $0 \quad(0.0)$ \\
\hline Trunk & $1(0.9)$ & $0 \quad(0.0)$ \\
\hline Extremities & $4(3.8)$ & $5 \quad(5.1)$ \\
\hline Other & $0 \quad(0.0)$ & $1(1.0)$ \\
\hline Length of laceration, $\mathrm{cm}$, mean $\pm \mathrm{SD}$ & $1.15 \pm 0.60$ & $1.31 \pm 0.68$ \\
\hline Age of laceration, hr, mean \pm SD & $1.93 \pm 3.02$ & $2.53 \pm 7.38$ \\
\hline $\begin{array}{l}\text { Length of time LET applied before } \\
\text { procedure, hr, mean } \pm \text { SD }\end{array}$ & $1.17 \pm 0.40$ & $1.21 \pm 0.94$ \\
\hline
\end{tabular}

and the interaction between categorized age and allocation were not significant $(p=0.4$ and $p=0.7$, respectively).

\section{Secondary outcomes}

Physicians more frequently rated wound hemostasis as complete in the treatment group (78.2\%) than in the placebo group $(59.3 \%)(p<0.008)$ (Table 3). The difference in physicians' rating of difficulty of wound repair between the 2 groups using the 100-mm Visual Analog Scale was not significant (median 6.50, IQR 2.5-17.5 for the treatment group; median 9.00, IQR 4-20 for the placebo group; Wilcoxon rank sum test $p=0.07$ ).

The results of a telephone survey at 2 weeks' follow-up showed no association between allocation and follow-up needs ( $n=139, p=0.7$ Fisher exact test) or families' subjective overall satisfaction with their children's wound repair $(n=139$, $p=0.5 \chi^{2}$ test). To assess the validity of the survey results and whether the surveyed subset was representative of all study participants, we compared the baseline characteristics of participants completing follow-up with those of the entire randomized group. Additional analyses did not show evidence to question the validity of the results (see Appendices 1, 2 and 3, available at www.cmaj.ca/lookup /suppl/doi:10.1503/cmaj.130269/-/DC1).

Physicians correctly guessed which treatment was applied in $72.8 \%$ of patients, with the analgesic incorrectly thought to be placebo in $10.4 \%$ of participants, and the placebo thought to be the analgesic in $16.8 \%$ of participants. Using Wilxocon tests, we found no differences between colour Visual Analogue Scale scores for patients in the treatment and control groups within those guessed to be in the control group $(p=0.7)$ and those guessed to be in the treatment group $(p=0.9)$ (Appendices 4 and 5, available at www.cmaj.ca /lookup/suppl/doi:10.1503/cmaj.130269/-/DC1).

\section{Interpretation}

\section{Main findings}

In this randomized, placebo-controlled trial of analgesic pretreatment of lacerations before tissue adhesive repair, we found pain reduction in pediatric patients receiving topically applied lidocaine-epinephrine-tetracaine.

Compared with children receiving placebo, children who received the analgesic had better wound hemostasis, fewer reports of pain and a clinically significant decrease in pain scores as assessed using the Faces Pain Scale - Revised, with no reported adverse effects when assessed by physicians in the emergency department or during the telephone survey at 2 weeks' follow-up.

The minimum clinically significant change 
reported for visual analog pain scales is $10 \%$ of the total scale. ${ }^{15,21}$ Thus, a statistically significant difference in medians of $0.5 \mathrm{~cm}$ on the colour Visual Analogue Scale does not achieve clinical relevance. The minimum clinically significant change in the Faces Pain Scale - Revised has been reported as 1 face..$^{15}$ Thus, the difference we saw on the Faces Pain Scale - Revised is clinically relevant and statistically significant.

Perhaps the most meaningful outcome for parents and children facing acute pain in the emergency department is how likely a procedure is to be completely pain free. More than half of the patients who received lidocaine-epinephrinetetracaine in our study (or their parents or guardians) reported no pain during adhesive application, which was nearly double the proportion of pain-free procedures reported in the placebo group.

Our findings are consistent with existing literature showing that tissue repair using an adhesive without anesthetic causes pain. ${ }^{2,3}$ We showed that this pain may be eliminated or reduced by the early topical application of lidocaine-epinephrinetetracaine. Singer and Stark showed that triage nurses could identify lacerations in children needing some form of primary closure with $95 \%$ specificity. ${ }^{23}$ Thus, our results suggest that triage nurses need not discern which lacerations require sutures versus tissue adhesive, as both could benefit from pretreatment with lidocaine-epinephrinetetracaine.

Priestley and colleagues found the application of lidocaine-epinephrine-tetracaine at triage reduced the total treatment time for children with minor lacerations (> 50\% repaired using tissue adhesive) by almost 30 minutes. ${ }^{24}$ Taken together with our finding that this analgesic reduces pain in tissue repairs using adhesive and improves the likelihood of a painless procedure, early application of lidocaine-epinephrine-tetracaine to all minor lacerations awaiting definitive physician repair could be a practical method of decreasing children's pain during these procedures.

\section{Limitations}

Physicians correctly guessed whether the analgesic or the placebo was applied $73 \%$ of the time, calling into question whether they were truly blinded to the treatment applied. Although efforts were made to construct this study as double-blinded by concealing treatment allocation from physicians, visible blanching on the skin of some patients occurs owing to the vasoconstricting effect of topically applied or locally administered epinephrine. However, all of the physicians who participated in our study completed a standardized, mandatory training mod- ule specifically instructing them not to give patients any indication concerning to which treatment arm they might have been allocated. In addition, nurses applying the intervention had no subsequent contact with the participants, and thus did not have the opportunity to see potential wound blanching. Finally, the patients themselves were unlikely to be aware of the possibility of skin blanching, and our primary outcome was patient reported.

Given physicians' statistically significant ability to correctly guess analgesic versus placebo, we examined descriptive statistics for colour Visual Analogue Scale scores for each combination of physicians' guesses (placebo or analgesic) and actual allocation (placebo or analgesic). We also used Wilcoxon tests to assess whether there were differences between actual allocations within guessed allocation and between guessed allocations within actual allocation.

When we compared baseline demographics between allocations within the excluded group and between the included and excluded groups in each allocated group, we did not find any obvious sources of bias.

We have presented the results of a perprotocol analysis. We considered imputation to allow an intention-to-treat analysis, but this analysis was not done because we determined that we could not produce reliable results.

Our study used only the particular formulation

Table 2: Pain as measured by scores on the colour Visual Analogue Scale and Faces Pain Scale - Revised for treatment and placebo groups

\begin{tabular}{|lccr|}
\hline & \multicolumn{2}{c}{ Score, median (IQR) } & \\
\cline { 2 - 3 } Pain scale & Treatment & Placebo & $p$ value* \\
\hline Colour Visual Analogue Scale & $0.50(0.25-1.50)$ & $1.00(0.38-2.50)$ & 0.01 \\
\hline Faces Pain Scale - Revised & $0.00(0.00-2.00)$ & $2.00(0.00-4.00)$ & $<0.01$ \\
\hline $\begin{array}{l}\text { Note: IQR }=\text { interquartile range. } \\
\text { *One-way } \chi^{2} \text { tests for equal proportions. }\end{array}$ & & \\
\hline
\end{tabular}

Table 3: Level of wound hemostasis achieved in treatment and placebo groups

\begin{tabular}{|lccc|}
\hline & \multicolumn{2}{c|}{ Study group, no. (\%) } & \\
\cline { 2 - 3 } Level of wound hemostasis & $\begin{array}{c}\text { Treatment } \\
n=101\end{array}$ & $\begin{array}{c}\text { Placebo } \\
n=91\end{array}$ & $p$ value* \\
\hline Complete, no bleeding & $79(78.2)$ & $54(59.3)$ & 0.008 \\
\hline Partial, mild oozing & $19(18.8)$ & $28(30.8)$ & 0.03 \\
\hline Poor, moderate oozing & $3(3.0)$ & $8(8.8)$ & 0.05 \\
\hline None, continuous bleeding & $0(0.0)$ & $1(1.1)$ & 0.2 \\
\hline $\begin{array}{l}\text { *Statistical difference was defined as } p<0.0125 \text { level using the Bonferroni correction due to } \\
\text { multiple comparisons. }\end{array}$ & & & \\
\hline
\end{tabular}


of tissue adhesive stocked in our hospital during the study period, $n$-butyl- 2 cyanoacrylate. The amount of pain felt during adhesive application is dependent on several factors, including the formulation, quantity and method of applying the adhesive. ${ }^{3,4}$ Quinn and Okun showed different peak temperatures and varying polymerization times in several different formulations of commercially available tissue adhesive using an aluminum foil model, ${ }^{25}$ although no in vivo difference in pain ratings between $n$-butyl- 2 cyanoacrylate and a popular octylcyanoacrylate formulation were found in a randomized trial comparing the two. ${ }^{20}$ Although the generalizability of our results to other tissue adhesives may be limited, lidocaine-epinephrinetetracaine is likely to have comparable effects with these products.

Our hospital pharmacy prepares 3-mL syringes of lidocaine-epinephrine-tetracaine from raw materials in-house at a cost of CAD $\$ 1.58$ per dose (including the cost of the actual syringe). There is also a "time cost" for patients, in that they must wait a minimum of 20 minutes for the analgesic to become effective, ${ }^{13}$ but this can be ameliorated by applying the solution while the patient is waiting for the physician. Although there are inherent difficulties in putting a dollar value on the benefit of pain reduction, it seems reasonable that many parents would feel these costs are justified to almost double the likelihood of their child feeling no pain during wound repair.

\section{Conclusion}

Pretreatment of minor lacerations with lidocaine-epinephrine-tetracaine before tissue repair with adhesive decreased patient discomfort and increased the proportion of pain-free repairs. Routine use of this simple and low-risk intervention as soon as the need for wound repair is identified may improve the experience of children undergoing treatment of lacerations. Future studies using other tissue adhesives and application methods, as well as investigation into potential cosmetic benefits, would help further define the merits of using lidocaine-epinephrine-tetracaine during tissue adhesive wound repairs.

\section{References}

1. Farion KJ, Russell KF, Osmond MH, et al. Tissue adhesives for traumatic lacerations in children and adults. Cochrane Database Syst Rev 2001;(4):CD003326.

2. Singer AJ, Giordano P, Fitch JL, et al. Evaluation of a new highviscosity octylcyanoacrylate tissue adhesive for laceration repair: a randomized, clinical trial. Acad Emerg Med 2003;10:1134-7.

3. Doraiswamy NV, Baig H, Hammett $\mathrm{S}$, et al. Which tissue adhesive for wounds? Injury 2003;34:564-7.

4. Bruns TB, Worthington JM. Using tissue adhesive for wound repair: a practical guide to dermabond. Am Fam Physician 2000; 61:1383-8.

5. Mattick A. Use of TSAs in the management of paediatric lacerations. Emerg Med J 2002;19:382-5.
6. Yamamoto LG. Preventing adeverse events and outcomes encountered using brand name tissue adhesive. Am J Emerg Med 2000;18:511-5156.

7. Cassidy R, Walco G. Pediatric pain: ethical issues and ethical management. Child Health Care 1996;25:253-64.

8. Weisman SJ, Bernstein B, Schechter NL. Consequences of inadequate analgesia during painful procedures in children. Arch Pediatr Adolesc Med 1998; 152:147-9.

9. Pate JT, Blount RL, Cohen LL, et al. Childhood medical experience and temperament as predictors of adult functioning in medical situations. Child Health Care 1996;25:281-98.

10. Eidelman A, Weiss JM, Baldwin CL, et al. Topical anaesthetics for repair of dermal laceration. Cochrane Database Syst Rev 2011; (6):CD005364.

11. Bhargava R, Young KD. Procedural pain management patterns in academic emergency pediatric departments. Acad Emerg Med 2007; 14:479-82.

12. Singer AJ, Kinariwala M, Lirov R, et al. Patterns of use of topic skin adhesives in the emergency department. Acad Emerg Med 2010;17:670-2.

13. Adler AJ, Dubinisky I, Eisen J. Does the use of topical lidocaine, epinephrine, and tetracaine solution provide sufficient anesthesia for laceration repair? Acad Emerg Med 1998;5:108-12.

14. White NJ, Kim MK, Brousseau DC, et al. The anesthetic effectiveness of lidocaine-adrenaline-tetracaine gel on finger lacerations. Pediatr Emerg Care 2004;20:812-5.

15. von Baeyer CL. Children's self-reports of pain intensity: scale selection, limitations and interpretation. Pain Res Manag 2006; 11:157-62.

16. McGrath PA, Seifert CE, Speechley KN, et al. A new analogue scale for assessing children's pain: an initial validation study. Pain 1996;64:435-43.

17. Hicks CL, von Baeyer CL, Spafford PA, et al. The faces pain scale - revised: toward a common metric in pediatric pain measurement. Pain 2001;93:173-83.

18. von Baeyer CL. Children's self-report of pain intensity: what we know, where we are headed. Pain Res Manag 2009;14:39-45.

19. Stinson JN, Kavanagh T, Yamada J, et al. Systematic review of the psychometric properties, interpretability and feasibility of self-report pain intensity measures for use in clinical trials in children and adolescents. Pain 2006;125:143-57.

20. Osmond MH, Quinn JV, Suitcliffe T, et al. A randomized, clinical trial comparing butylcyanoacrylate with octylcyanoacrylate in the management of selected pediatric facial lacerations. Acad Emerg Med 1999;6:171-7.

21. Kelly A-M. The minimum clinically significant difference in visual analogue scale pain score does not differ with severity of pain. Emerg Med J 2001;18:205-7.

22. Dunn OJ. Multiple comparisons among means. J Am Stat Assoc 1961;56:52-64.

23. Singer AJ, Stark M. Pretreatment of lacerations with lidocaine, epinephrine, and tetracaine at triage: a randomized double-blind trial. Acad Emerg Med 2000;7:751-6.

24. Priestley S, Kelly AM, Chow L, et al. Application of topical local anesthetic at triage reduces treatment time for children with lacerations: a randomized controlled trial. Ann Emerg Med 2003; 42:34-40.

25. Quinn JV, Okun N. Exothermic effects of cyanoacrylate tissue adhesives [abstract]. Acad Emerg Med 2006;13:S79.

Affiliations: Division of Emergency Medicine (Harman, Zemek), Department of Pediatrics; Division of Plastic Surgery (Duncan, Ying), Department of Surgery, University of Ottawa; Methods Centre (Petrcich), Ottawa Hospital Research Institute; and the Children's Hospital of Eastern Ontario (Harman, Zemek, Duncan, Ying), Ottawa, Ont.

Contributors: Stuart Harman, Roger Zemek, Mary Jean Duncan and Yvonne Ying conceived and designed the study and acquired the data. Stuart Harman, Roger Zemek and William Petrcich analyzed and interpreted the data and drafted the manuscript. All of the authors critically revised the manuscript for important intellectual content. All of the authors approved the final version of the manuscript submitted for publication.

Funding: Funded by the Children's Hospital of Eastern Ontario Research Institute. The study sponsors had no role in the design of the study, the collection, analysis or interpretation of data, the writing of the report or the decision to submit the article for publication. 